\title{
Implementing Optogenetic Modulation in Mechanotransduction
}

\author{
Miao Yu, ${ }^{1,2, *}$ Shimin Le, ${ }^{2, *}$ Samuel Barnett@ ${ }^{1, *}$ Zhenhuan Guo, ${ }^{1}$ Xueying Zhong, ${ }^{1}$ \\ Pakorn Kanchanawong, ${ }^{1,3,}$ and Jie Yan $\circledast^{1,2, \$}$ \\ ${ }^{1}$ Mechanobiology Institute, National University of Singapore, 117411 Singapore \\ ${ }^{2}$ Department of Physics, National University of Singapore, 117542 Singapore \\ ${ }^{3}$ Department of Biomedical Engineering, National University of Singapore, 117583 Singapore
}

(Received 10 July 2019; revised manuscript received 9 January 2020; accepted 26 February 2020; published 1 April 2020)

\begin{abstract}
Molecular optogenetic switch systems are extensively employed as a powerful tool to spatially and temporally modulate a variety of signal transduction processes in cells. However, the applications of such systems in mechanotransduction processes where the mechanosensing proteins are subject to mechanical forces of several piconewtons are poorly explored. In order to apply molecular optogenetic switch systems to mechanobiological studies, it is crucial to understand their mechanical stabilities which have yet to be quantified. In this work, we quantify a frequently used molecular optogenetic switch, iLID-nano, which is an improved light-induced dimerization between LOV2-SsrA and SspB. Our results show that the iLID-nano system can withstand forces up to $10 \mathrm{pN}$ for seconds to tens of seconds that decrease as the force increases. The mechanical stability of the system suggests that it can be employed to modulate mechanotransduction processes that involve similar force ranges. We demonstrate the use of this system to control talin-mediated cell spreading and migration. Together, we establish the physical basis for utilizing the iLID-nano system in the direct control of intramolecular force transmission in cells during mechanotransduction processes.
\end{abstract}

DOI: 10.1103/PhysRevX.10.021001

Subject Areas: Biological Physics

\section{INTRODUCTION}

Mechanotransduction is a critical physiological process in which cells sense physical forces and translate them into biochemical responses. The molecular basis of mechanotransduction comprises various mechanosensitive proteins whose interactions are mediated by the physiological level of forces over a physiological timescale [1,2]. For instance, cell adhesion, spreading, and migration on the extracellular matrix (ECM) rely on a large set of focal adhesion proteins, such as integrin, talin, vinculin, paxillin, etc., [3] [Fig. 1(a)]. To decode the molecular mechanisms of mechanotransduction mediated by these proteins, it is critical to be able to temporally and spatially control specific force transmission through specific supramolecular linkages. During testing of the ECM stiffness, the cell leading edge protrudes and contracts with a displacement on the order of a few

\footnotetext{
*These authors contributed equally to this work.

${ }^{\dagger}$ To whom the correspondence should be addressed. biekp@nus.edu.sg

${ }^{*}$ To whom the correspondence should be addressed. phyyj@nus.edu.sg

Published by the American Physical Society under the terms of the Creative Commons Attribution 4.0 International license. Further distribution of this work must maintain attribution to the author(s) and the published article's title, journal citation, and DOI.
}

hundreds of nanometers [4]. These oscillations occur with a typical timescale of tens of seconds, and the forces through individual force-transmission linkages at focal adhesions are typically between approximately 2 and 10 piconewtons $(\mathrm{pN})[5,6]$. Thus, the physiological pulling rate is on the order of tens of $\mathrm{nm} \mathrm{s}^{-1}$ and the force-loading rate on the order of a few $\mathrm{pN} \mathrm{s}^{-1}$.

A force-transmission supramolecular linkage typically consists of several noncovalently linked proteins that contain a tandem of force-bearing structural domains and intermolecular interfaces. The lifetimes of the intermolecular interfaces at the physiological level of forces are on the order of seconds to hundreds of seconds depending on the level of force and the specific intermolecular interfaces [10-12]. As a result from the limited lifetime of the intermolecular interfaces, the force-bearing linkages undergo dynamic assembly and disassembly. For several linkages at focal adhesion and cell-cell adherence junctions, the differential mechanical stability of the intermolecular interfaces results in a typical linkage lifetime ranging from seconds to tens of seconds estimated using fluorescence recovery after photobleaching (FRAP) assays $[13,14]$.

In addition, many of the structural domains in a forcetransmission pathway undergo dynamic unfolding and refolding over a force range from several to tens of piconewtons at physiological pulling or loading rates 
(a)
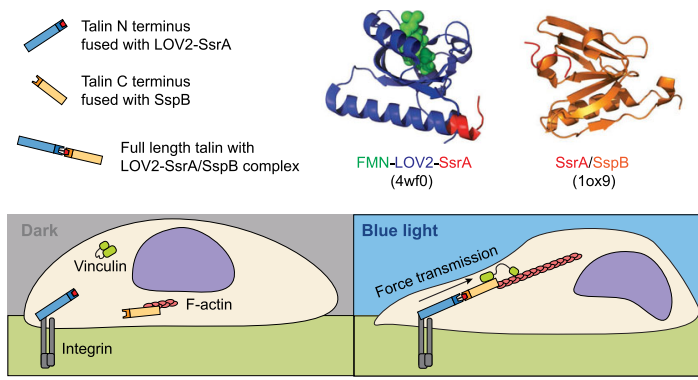

(b)

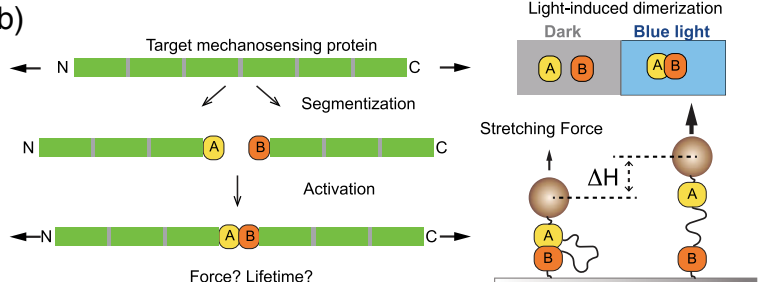

(c)
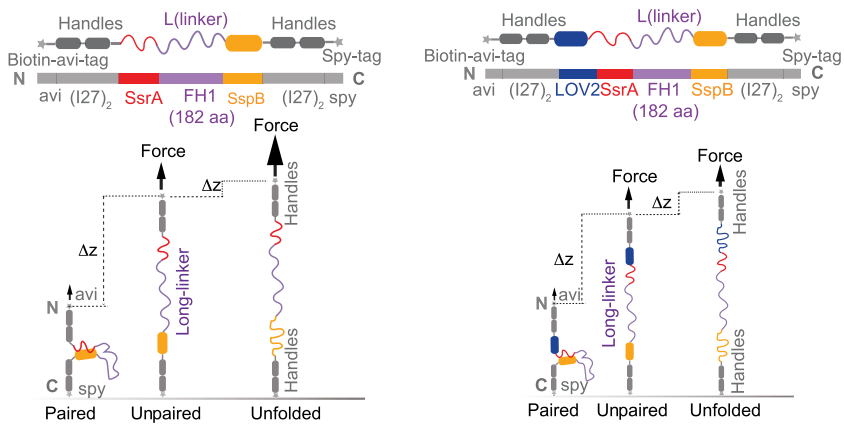

FIG. 1. Illustration of potential implementation of optogenetic modulation. (a) Illustration of a talin-mediated mechanotransduction linkage in a cell. The truncated talin molecule segments (talin $\mathrm{N}$ terminus and talin $\mathrm{C}$ terminus) are fused with the dimerizing domains LOV2-SsrA and SspB, respectively (top left). The crystal structures of LOV2-SsrA (PDB: 4wf0) and SsrA-SspB (PDB: 1ox9) are also shown (top right) [7,8]. (b). Left: Illustration of the potential implementation of the molecular optogenetic switch systems to connect the two segments of a truncated protein. Right: Illustration of the experimental design of the single-molecule stretching on the light-sensitive dimers. (c). Experimental design of single-molecule SsrA/SspB (left) and LOV2-SsrA/SspB (right) constructs. Top: Single-molecule constructs (N-to-C terminus): avi-tag for biotinylation, two repeats of the titin Ig 27th domain (I27), SsrA (left) or LOV2-SsrA (right), a long flexible unstructured peptide chain FH1 domain, SspB, two repeats of I27, and spy-tag [9] for the specific spy-catcher attachment. Bottom: A single-molecule construct is specifically tethered between a cover slip surface and a magnetic bead with an approximately 600-bp DNA handle (more details in Sec. IV).

[15-18]. This process makes them an effective force buffer to maintain the tension in the force-transmission pathway at a similar range $[15,17]$. The physiologically relevant force range predicted based on the force-dependent unfolding and refolding of structural domains is confirmed with direct tension measurement in live cells using the recently developed fluorescence resonance energy transfer tension sensors for several important force-transmission pathways at focal adhesion and cell-cell adherence junctions [5,6,19]. The lifetime of talin in focal adhesions is estimated using a FRAP assay on the order of a few tens of seconds $[13,14]$. As force-transmission linkages are the physical scaffolds to mediate specific mechanotransduction processes, they provide a potential handle to modulate mechanotransduction through controlling specifically targeted force-transmission linkages.

We explore the possibility of controlling the force transmission in targeted force-transmission linkages using molecular optogenetic switch systems, also termed as optical dimerizers. These systems are employed as powerful tools to influence signal transduction or transport [7,20-24]. Molecular optogenetic switch systems are also implemented in modulating mechanotransduction [25,26] through directing upstream master signaling proteins related to mechanotransduction, such as RhoA activator, to specific locations in cells. All these previous applications are based on controlling the vicinity of signaling proteins to their interacting partners where the targeted molecules are not subject to mechanical forces. Furthermore, upstream signaling master controls of mechanotransduction tend to activate multiple molecular processes which may obfuscate molecular-level mechanistic dissection.

To apply molecular optogenetic switch systems to mechanotransduction processes where the mechanosensing proteins are subject to mechanical forces, an important prerequisite is that the switch systems are able to withstand the physiological level of forces for physiologically relevant timescales. The mechanical stability of the switch systems is poorly understood. Only very recently, the stability of a system, pdDronpa1.2 homodimer, was shown able to withstand approximately $80 \mathrm{pN}$ using atomic force microscopy at a pulling rate of $1600 \mathrm{~nm} \mathrm{~s}^{-1}$ [27]. This pulling rate is orders of magnitude higher than the typical pulling rates applied to mechanosensing proteins in cells, which is on the order of a few tens of nanometers per second [4]. To our knowledge, the mechanical stability of molecular optogenetic switch systems at physiologically relevant pulling or loading rates has not been investigated.

We seek to directly quantify the mechanical stability of a molecular optogenetic switch system and explore its possible implementation in mechanotransduction studies [Figs. 1(a) and 1(b), left]. We choose iLID-nano [7], a widely used light-induced dimerization system for characterization. Briefly, the iLID-nano system involves two components, a 114 a.a. SspB domain from Escherichia coli and LOV2-SsrA comprising a short peptide SsrA (8 a.a.) integrated into the light-sensitive domain LOV2 (143 a.a.) from the plant Avena sativa [7,8] [Fig. 1(a)]. In the absence of blue-light activation (dark state), the LOV2 domain interacts with SsrA, thereby suppressing the interaction between SsrA and SspB. In the presence of activating blue light (light-activated state), the autoinhibitory 
interaction between LOV2 and SsrA is relieved, thereby exposing SsrA for interaction with SspB. A key cofactor for the light sensitivity of the LOV2 domain is a flavin molecule bound in the domain core [28-31]. Blue-light absorption promotes the noncovalently bound flavin to a covalent adduct state, which then thermally decays to regenerate a dark state with a lifetime of approximately $80 \mathrm{~s}$ for the A. sativa LOV2 domain [29,31]. Hence, the iLID-nano system may have three relatively stable states: (i) an "apo state" where the LOV2 domain is not bound by flavin, (ii) a "dark state" where the LOV2 domain is bound with a flavin in the absence of blue-light activation, and (iii) an "active state" where the flavin-bound LOV2 domain is activated by blue light.

In this study, we characterize the mechanical signature of LOV2 in the presence and absence of a bound flavin mononucleotide (FMN) and show that FMN binding strongly stabilizes the LOV2 domain. We quantify the effect of FMN binding on the autoinhibition strength of LOV2 that suppresses the SsrA-SspB interaction. We show that the paired iLID-nano system where the LOV2-SsrA/ $\mathrm{SspB}$ complex forms can withstand forces up to $10 \mathrm{pN}$ for seconds to tens of seconds, which is independent of the light condition or whether FMN is bound on LOV2. The mechanical stability of the iLID-nano system suggests that it can be employed to modulate mechanotransduction processes that involve similar force ranges, which is demonstrated by utilizing the system to control talinmediated cell spreading and migration.

\section{RESULTS}

To investigate whether the iLID-nano system meets the requirement of mechanotransduction studies, i.e., capable of withstanding a physiological force range in a forcetransmission supramolecular linkage associated with a mechanotransduction process over a physiological timescale, we develop a single-molecule-construct assay to directly quantify the mechanical lifetime of the iLID-nano system.

\section{A. Single-molecule construct of the molecular optogenetic switch system}

The single-molecule construct comprises two lightsensitive interacting partners, $A$ and $B$, connected by a 182 a.a. flexible unstructured peptide linker [32] [Fig. 1(b), right]. The construct is tethered between a cover slip and a 2.8- $\mu$ m-diameter superparamagnetic bead and is stretched by forces applied using an in-house-built magnetic tweezers setup [33-36]. The construct can exist in a paired state where $A$ and $B$ form a complex, $A / B$, or an unpaired state where $A$ and $B$ are separated.

Because of the long flexible linker, the paired and unpaired states of the construct have a significant extension difference that can be detected by the magnetic tweezers setup. Therefore, when the applied force is low, $A$ and $B$ are able to form a complex, and the tether is shorter. As the applied force increases to a point where the complex is disrupted, one observes stepwise extension increases due to disruption of the complex and extension of the flexible linker and potentially unfolding of the structural domains of $A$ or $B$ in the construct. When the applied force returns to low levels, the construct repairs when $A$ and $B$ reform the complex and allows the construct to be unpaired in the next force-increase scan. Therefore, the construct can undergo multiple unpairing and repairing cycles during the forceincrease and force-decrease scan cycles, respectively. By directly measuring the lifetime of the construct before its unpairing at various forces, we can quantify the mechanical lifetimes of the molecular optogenetic switch system.

\section{B. Force response of the SsrA/SspB complex}

To apply the method to investigate the mechanical stability of the iLID-nano system that involves two interacting partners LOV2-SsrA and SspB, we first quantify the stability of the SsrA and SspB interaction in the absence of a light-sensitive autoinhibitory LOV2 domain [Fig. 1(c), left]. Since the construct does not contain the light-sensitive LOV2 domain, the experiments are carried out in ambient light conditions. Typical force-extension curves of the SsrA/SspB complex obtained by increasing the force from approximately $1 \mathrm{pN}$ at a force-loading rate of $0.5 \pm$ $0.1 \mathrm{pN} \mathrm{s}^{-1}$ to approximately $20 \mathrm{pN}$ are shown in Fig. 2(a). One or two stepwise extension increases are observed, indicating the existence of two transition events. The step size involved in the single-step extension increase is the sum of the step sizes of the dual-step extension increases; therefore, the single-step extension increase can be considered as two coincident transition events.

In the SsrA/SspB construct, the possible transitions are the unpairing of the SsrA/SspB complex and the following unfolding of SspB. Since SsrA is a short peptide, it cannot contribute to such stepwise extension increase signals. The number of released residues involved in the extension increase signal is estimated to be $248 \pm 91(5)$ a.a. [mean \pm s.t.d. (s.e.), Fig. S1 [37]], based on the forcedependent step size of the unlooping transition and the force-extension curve of the polypeptide assuming the wormlike chain polymer model with a bending persistence length of approximately $0.8 \mathrm{~nm}[32,38]$ (Fig. S1, Supplemental Material Sec. S2 [37]). This value is consistent with releasing the looped long flexible linker in the construct. In addition, the estimated number of residues during the unfolding transition of $\mathrm{SspB}$ is $113 \pm 26(2)$ a.a. (Fig. S1 [37]), which is also consistent with the number of residues of the $\mathrm{SspB}$ domain.

The normalized histogram of approximately 300 unpairing forces obtained from five different tethered molecules shows a two-peak profile, which can be fitted with doubleGaussian distribution [Fig. 2(b)]. The result suggests the existence of two distinct mechanical stability groups of the 

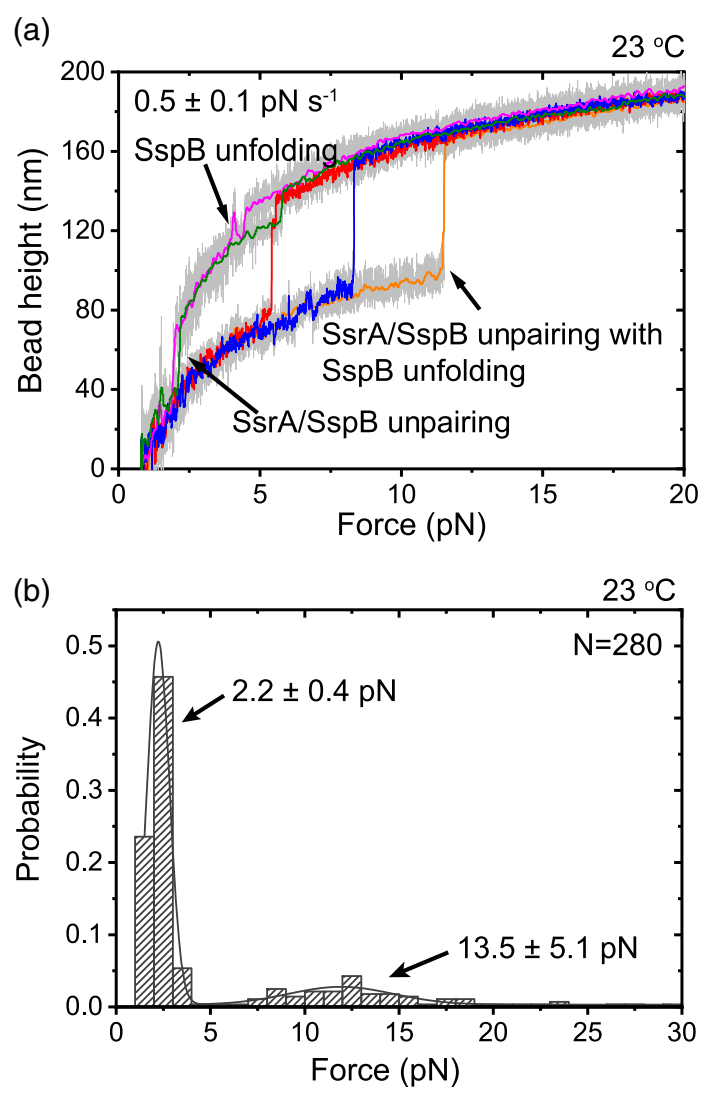

FIG. 2. Mechanical stability of the SsrA/SspB complex. (a) Five representative force-extension curves of the SsrA-SspB construct obtained at a force-loading rate of $0.5 \pm 0.1 \mathrm{pN} \mathrm{s}^{-1}$ under ambient light conditions. The color lines show a 20-FFT smooth of the raw data (gray). (b) Normalized histograms of the SsrA/ $\mathrm{SspB}$ complex unpairing forces at a force-loading rate of $0.5 \pm 0.1 \mathrm{pN} \mathrm{s}^{-1} . N$ indicates the number of data points obtained. The dark gray curve is a double Gaussian fitting curve.

SsrA/SspB complex, which are unpaired at $2.2 \pm 0.4(0.03)$ and $13.5 \pm 5.1(0.6) \mathrm{pN}$, respectively. In addition, the $\mathrm{SspB}$ domain unfolds at $5.3 \pm 1.7(0.1) \mathrm{pN}$ (Fig. S1 [37]). Here, the errors of the forces are standard deviations of the distribution and standard errors of the mean in the brackets. An interesting observation is that the two mechanical stability groups appear in a manner clustered in the time trace during repeating force scan cycles, which could persist for tens of cycles until switching from one group to another (Figs. S2 and S8, dark gray [37]). Because of the long persistent time of the clustered events, the accurate determination of the probability ratio between the two groups may require a large number of cycles that is beyond our experimental timescale. However, the results clearly demonstrate the existence of two mechanical stability groups, both with significant fractions.

\section{Force response of the LOV2-SsrA/SspB complex}

We next seek to probe the force responses of the LOV2SsrA/SspB complex using the LOV2-SsrA/SspB construct
[Fig. 1(c), right]. Since FMN is noncovalently bound to LOV2 in the dark state, FMN could dynamically dissociate from and reassociate to LOV2 spontaneously, which is facilitated by chemical denaturation of the LOV2 domain [29-31]. Hence, it is reasonable to predict that the forceinduced unfolding of the LOV2 domain (i.e., force-induced domain denaturation) may facilitate the dissociation and association of FMN. Together with the externally controlled light condition, force applied to the LOV2-SsrA/ SspB complex enables controlling the FMN-binding states of LOV2, the structural states of LOV2 and SspB in the iLID-nano system, and the pairing state between LOV2-SsrA and SspB, as well as the blue-light activation of the iLID system in our experiments. We perform the single-molecule stretching experiments in four different conditions: (i) in red light with $2 \mu \mathrm{M}$ FMN in solution, (ii) in red light without FMN in solution, (iii) in blue light with $2 \mu \mathrm{M}$ FMN in solution, and (iv) in blue light without FMN in solution. Under these experimental conditions, the iLID-nano system could exist in different FMN-binding, structural, and pairing states.

Figures 3(a) and 3(b) show typical force-bead height curves of the LOV2-SsrA/SspB construct with red light [(a) 617-nm red LED] or blue light [(b) 470-nm blue LED], respectively, in the presence of $2 \mu \mathrm{M}$ FMN in solution, obtained during increasing the force from approximately $1 \mathrm{pN}$ to approximately $60 \mathrm{pN}$ with a loading rate of $0.5 \pm 0.1 \mathrm{pN} \mathrm{s}^{-1}$. The construct is held for approximately $20 \mathrm{~s}$ at low forces of approximately $0.3 \mathrm{pN}$ prior to each force-increase scan for the formation of the LOV2-SsrA/ $\mathrm{SspB}$ complex. The resulting unpairing and unfolding force distributions of the construct under different lights at corresponding loading rates are plotted in Figs. 3(c), 3(d), S3, and S4 [37].

In red light, the majority $(>80 \%)$ of the force-increase scans show only two stepwise extension increase transitions: One can be attributed to $\mathrm{SspB}$ unfolding that typically occurs below approximately $6 \mathrm{pN}$ characterized by releasing of $120 \pm 25(1)$ a.a. residues, and the other occurring at $>10 \mathrm{pN}$ can be attributed to LOV2 unfolding characterized by releasing of $125 \pm 22(5)$ a.a. residues [Figs. 3(c) and S3 [37]]. In these scans, no characteristic unpairing signals of the LOV2-SsrA/SspB complex, which should involve much larger step sizes at these transitions forces, are observed. In the remaining $<20 \%$ forceincrease scans, the characteristic unpairing transitions that occur at approximately $8 \mathrm{pN}$ associated with the release of $238 \pm 31$ (3) a.a. residues are observed (Fig. S3 [37]). In sharp contrast, in blue light, the majority $(>70 \%)$ of the force-scan cycles show the characteristic unpairing transition [release of $273 \pm 70(5)$ a.a. at $6.7 \pm 2.4(0.2) \mathrm{pN}$ ], in addition to unfolding of SspB [release of $111 \pm 24(2)$ a.a. at $5.1 \pm 1.5(0.1) \mathrm{pN}$ ] and LOV2 [release of $108 \pm 27(3)$ a.a. at $16.8 \pm 0.5(0.1) \mathrm{pN}$ and at $43.9 \pm 3.1(0.4) \mathrm{pN}]$ [Figs. 3(c), 3(d), and S4 [37] ]. 

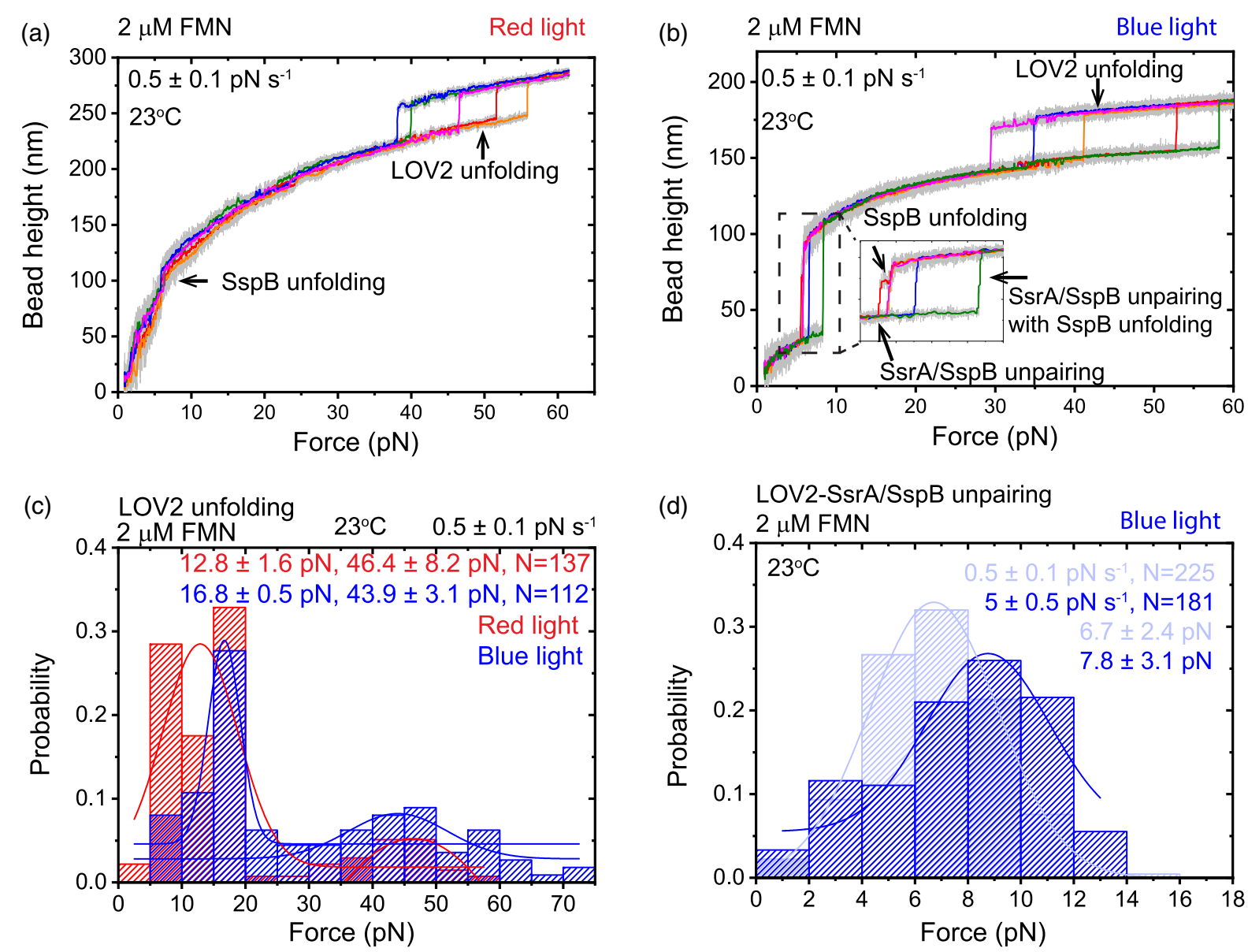

FIG. 3. Mechanical stability of the FMN-LOV2-SsrA/SspB complex. (a),(b) Five representative force-extension curves of the FMNLOV2-SsrA/SspB complex obtained at a force-loading rate of $0.5 \pm 0.1 \mathrm{pN} \mathrm{s}^{-1}$ with $2 \mu \mathrm{M}$ free FMN in solution, in red light and in blue light [collimated blue LED array (approximately $1 \mathrm{~mW} \times \mathrm{cm}^{-2}$ at $470 \mathrm{~nm}$ ) [7] ], respectively, indicated by multiple colors. The color lines are 20-FFT smooth of the raw data (gray). (c) Normalized histograms of LOV2 unfolding forces obtained in red light (red)and blue light (blue), with $2 \mu \mathrm{M}$ free FMN in solution. (d) Normalized histograms of FMN-LOV2-SsrA/SspB complex unpairing forces obtained in blue light at force-loading rate of $0.5 \pm 0.1$ and $5 \pm 0.5 \mathrm{pN} \mathrm{s}^{-1} . N$ indicates the number of data points obtained. The colored lines in (c) and (d) are corresponding Gaussian fitting curves.

Interestingly, the LOV2 unfolding force distribution shows two peaks, one at approximately $13 \mathrm{pN}$ and the other at approximately $45 \mathrm{pN}$. We reason that such a twopeak unfolding force distribution may correspond to different FMN-binding states of LOV2 (i.e., the apo and the FMN-bound states). To test this hypothesis, we investigate the force response of the iLID-nano system in the absence of FMN in solution. Under such conditions, the FMN might dissociate from LOV2, generating the apo state of LOV2.

Figures 4(a) and 4(b) show typical force-bead height curves of the LOV2-SsrA/SspB construct in FMN-free solution conditions with red light (a) or blue light (b) at a loading rate of $0.5 \pm 0.1 \mathrm{pN} \mathrm{s}^{-1}$, respectively. The unpairing and unfolding force distributions of the construct at corresponding loading rates are plotted in Figs. 4(c), 4(d), S5, and S6 [37]. Interestingly, the LOV2 unfolding forces distribute around a single peak at approximately $13 \mathrm{pN}$ [Fig. 4(c)], similar to the first peak of the LOV2 unfolding force distribution obtained in the presence of $2 \mu \mathrm{M}$ FMN [Fig. 3(c)]. Together, these results strongly suggest that, in the presence of $2 \mu \mathrm{M}$ FMN, the first and second peaks of the LOV2 unfolding force distribution correspond to the unfolding of LOV2 in the apo state and the FMN-bound state, respectively. Such FMN-binding-mediated mechanical stabilization of LOV2 can be considered as a signature for the FMN-bound state of LOV2 and utilized for the detection of FMN binding to LOV2.

In addition, with both red- and blue-light illumination, the unpairing forces of the LOV2-SsrA/SspB complex exhibits a single-peak Gaussian-like distribution with a similar peak force of 5.5-7.8 pN [Figs. 3(d), S3 [37], and 4(d)]. This result is in contrast to the unpairing of the SsrA/ SspB complex, which shows two distinct mechanical stability groups [Fig. 2(b)]. We reason that the presence of LOV2 might impose a constraint on the possible conformation of the paired $\mathrm{SsrA}$ and $\mathrm{SspB}$, or the 

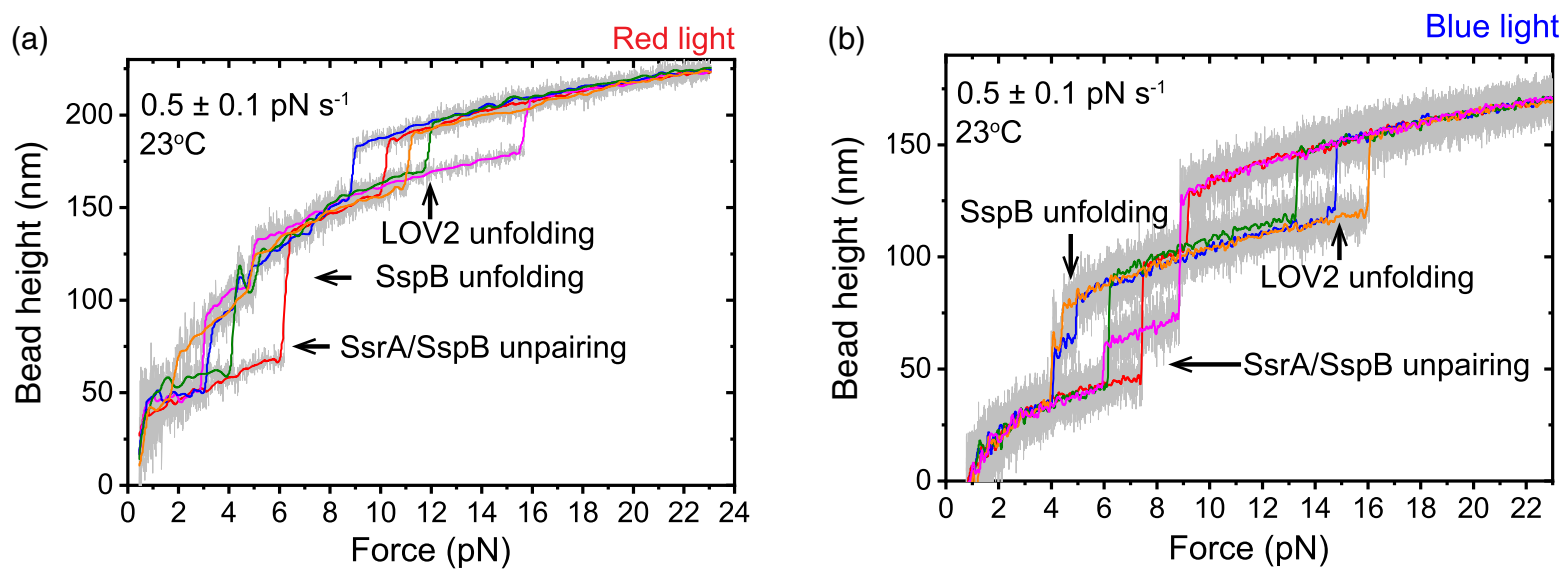

(c)
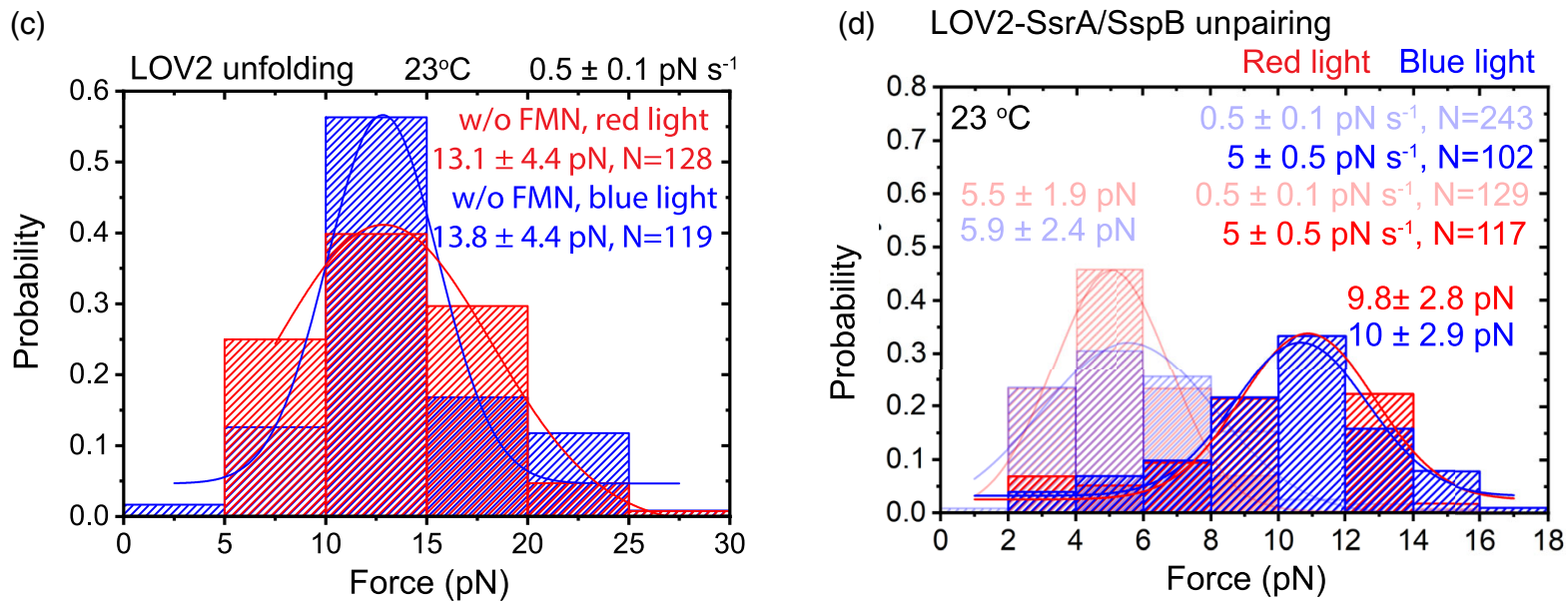

FIG. 4. Mechanical stability of the apo LOV2-SsrA/SspB complex. (a),(b) Five representative force-extension curves of the apo LOV2-SsrA/SspB complex obtained in red light and in blue light, respectively, at a force-loading rate of $0.5 \pm 0.1 \mathrm{pN} \mathrm{s}^{-1}$, indicated by multiple colors. The colored lines are 20-FFT smooth of the raw data (gray). (c) Normalized histograms of apo LOV2 unfolding forces with a loading rate of $0.5 \pm 0.1 \mathrm{pN} \mathrm{s}^{-1}$ obtained in red light (red) and blue light (blue), respectively. $N$ indicates the number of data points. The colored lines in (c) and (d) are corresponding Gaussian fitting curves. (d) Normalized histograms of apo LOV2-SsrA/SspB complex unpairing forces at a force-loading rate of $0.5 \pm 0.1$ and $5 \pm 0.5 \mathrm{pN} \mathrm{s}^{-1}$ obtained in red light (red) and blue light (blue), respectively.

accessible transition pathways during mechanical unpairing of the LOV2-SsrA/SspB complex, resulting in the singlepeak unpairing force distribution.

\section{Light-sensitive autoinhibition of LOV2 on the SsrA-SspB interaction}

Since the central design element of the iLID system is the autoinhibition of the formation of the LOV2-SsrA/SspB complex by the light-sensitive interaction between LOV2 and SsrA, we next probe the effects of the autoinhibitory strength of the LOV2 domain, by performing a low-forcewaiting assay on the LOV2-SsrA/SspB single-molecule construct. Briefly, a LOV2-SsrA/SspB construct [Fig. 1(c), right] is held at low forces of approximately $0.3 \mathrm{pN}$ for various waiting times in the range of $10-40 \mathrm{~s}$, to allow the potential formation of a complex. Then the force is increased linearly to check whether the complex forms during the low-force-waiting time period based on the characteristic unpairing signal [Fig. 5(a)] and whether the LOV2 is bound with FMN based on whether LOV2 remains folded when forces reach $22 \mathrm{pN}$ (note that apo LOV2 unfolds at approximately $13 \mathrm{pN}$ ). Repeating the lowforce-waiting procedure for over 80 cycles from more than five different tethers, we obtain the probability of the FMNLOV2-SsrA/SspB complex formation at each lowforce-waiting time for the single-molecule construct at corresponding experimental conditions [Fig. 5(b)].

With $2 \mu \mathrm{M}$ FMN in solution in red light, the FMNbound LOV2-SsrA/SspB construct has a probability of $16 \%-21 \%$ of forming the LOV2-SsrA/SspB complex for a waiting time over a range of $10-40 \mathrm{~s}$ in a waiting-timeindependent manner [red data in Fig. 5(b)], consistent with a certain level of dark state activity of the iLID-nano system [7]. In contrast, under the experimental condition, the SsrA/ SspB construct [Fig. 1(c), left] without LOV2 forms the 


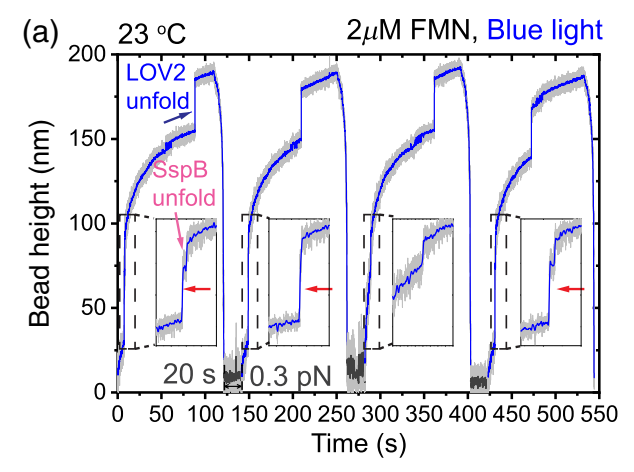

(b)
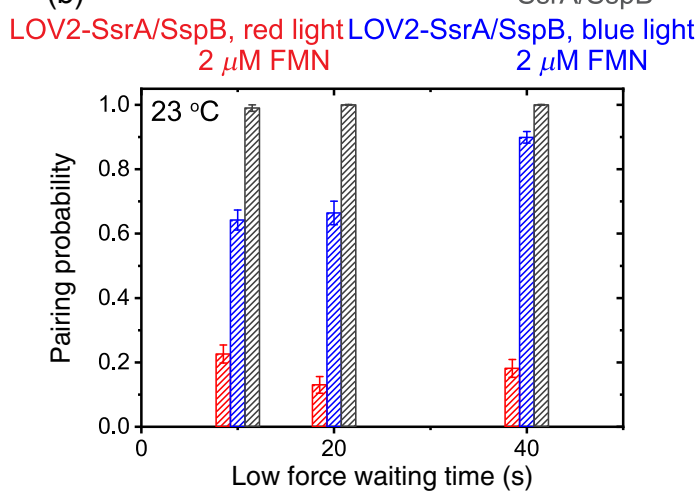

FIG. 5. Autoinhibition strength of LOV2 on the SsrA-SspB interaction. (a) Representative time trace of bead height change during a force-loading assay to probe the effects of the autoinhibitory strength of the LOV2 domain on the SsrA-SspB interaction. In this example, after holding the LOV2-SsrA/SspB construct at $0.3 \mathrm{pN}$ for $20 \mathrm{~s}$ in blue light with $2 \mu \mathrm{M}$ FMN, the force is increased at a loading rate of $0.5 \pm 0.1 \mathrm{pN} \mathrm{s}^{-1}$ to approximately $60 \mathrm{pN}$. The LOV2-SsrA/SspB complex is formed in three of four force-loading cycles indicated by unpairing signals in the subsequent force-increase step (red arrows in the enlarged insets). The blue and dark gray lines show a 20-FFT smooth of the raw data (gray). (b) The probabilities of formation of the FMN-LOV2-SsrA/SspB complex with $2 \mu \mathrm{M}$ FMN in red light (red) and in blue light (blue) within different holding time intervals at approximately $0.3 \mathrm{pN}$. The formation probability of the SsrA/SspB complex (gray) is plotted for comparison. For each condition, over 80 force cycles are performed for statistical analysis. The error bars indicate the standard deviation obtained from a bootstrap analysis (Supplemental Material, Sec. S3 [37]).

SsrA/SspB complex with $>97 \%$ probability [Fig. 5(b), gray]. The large difference in the pairing probability between the FMN-bound LOV2-SsrA/SspB construct and the SsrA/SspB construct is consistent with previous biochemical studies that reveal the autoinhibitory role of the FMN-bound LOV2 domain [30,31].

Furthermore, repeating the same experiment for the LOV2-SsrA/SspB construct in $2 \mu \mathrm{M}$ FMN under blue light, the pairing probability increases to $62 \%-88 \%$ for waiting times over a range of 10-40 s [blue data in Fig. 5(b)]. Compared to $<20 \%$ pairing probability under red-light conditions, this result is consistent with blue light weakening the autoinhibitory interaction of FMN-bound
LOV2 and SsrA, activating the SsrA-SspB interaction. In addition, repeating the same experiments in the absence of FMN, the results show that the pairing probability exceeds $80 \%$ at $\geq 40$ s holding time at $0.3 \mathrm{pN}$, in both blue- and redlight conditions (Fig. S7 [37]). The results suggest that the apo state of LOV2 loses its autoinhibition activity.

\section{E. Mechanical lifetime of the paired iLID-nano system}

To test the suitability of using the iLID-nano system to control cellular mechanotransduction, we examine the mechanical lifetime of the LOV2-SsrA/SspB complex [Fig. 1(c), right]. We measure the mechanical lifetimes of the complex at different forces using a force clamp-cycle procedure. Briefly, in each cycle, a construct is first held at low forces of approximately $0.3 \mathrm{pN}$ for $20 \mathrm{~s}$ to allow the formation of the complex and then held at different forces in the range of $2-15 \mathrm{pN}$ until the unpairing transition is observed [Fig. 6(a)]. By repeating the force-clamp cycles over 80 times for each force, the force-dependent lifetimes of the corresponding complex at the pairing state are obtained. Figure 6(b) shows the obtained force-dependent lifetimes of the LOV2-SsrA/SspB complex in red light without FMN in solution (light red), LOV2-SsrA/SspB complex in blue light without FMN in solution (light blue), and LOV2-SsrA/SspB complex in blue light with $2 \mu \mathrm{M}$ FMN (blue).

Clearly, with either red- or blue-light illumination, the LOV2-SsrA/SspB complex can withstand forces up to $10 \mathrm{pN}$ over seconds to tens of seconds, and the FMNbound LOV2 or apo-LOV2 does not affect the mechanical lifetime of the LOV2-SsrA/SspB complex. Together, these results suggest that both the light and the LOV2 domain mainly regulate the autoinhibitory states of the iLID-nano system. However, they do not affect the mechanical stability of the paired system. These forces and lifetime values are similar to the physiological force and lifetime ranges of several force-transmission supramolecular linkages involved in mechanotransduction at focal adhesion and cell-cell adherence junctions $[5,6,13,15]$, suggesting that the iLID-nano system can potentially be implemented for the studies of these mechanotransduction processes.

\section{F. Modulation of talin-mediated mechanotransduction using the iLID-nano system}

In previous sections, we show that the LOV2-SsrA/SspB complex is capable of withstanding several piconewton forces over a lifetime of seconds to tens of seconds at $23^{\circ} \mathrm{C}$, which is independent of FMN binding and light conditions. To implement the iLID-nano system to control mechanotransduction of living cells at a human body temperature of $37^{\circ} \mathrm{C}$, we probe the force responses of the LOV2-SsrA/ SspB complex at different loading rates [Figs. 7(a) and S9 [37] ] and the mechanical lifetime of LOV2-SsrA/SspB complex [Fig. 7(b)] at $37^{\circ} \mathrm{C}$. The results show that the mechanical stability of the LOV2-SsrA/SspB complex 

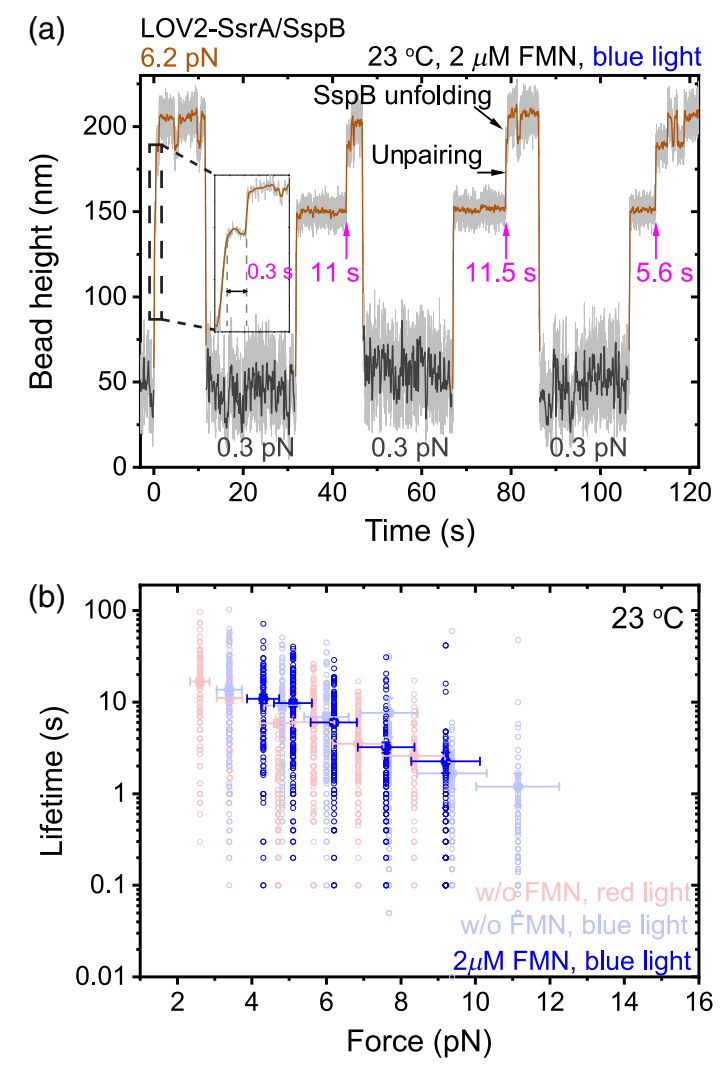

FIG. 6. Mechanical lifetime of the LOV2-SsrA/SspB complexes. (a) Representative time trace of bead height change during a force-clamp assay to quantify the lifetime of the LOV2-SsrA/ $\mathrm{SspB}$ complex at various forces. After jumping from $0.3 \mathrm{pN}$ to a higher force, the average lifetime of the complex at the higher force is measured in multiple cycles. In this example, the lifetime of the complex at $6.2 \mathrm{pN}$ in four force jumps is shown. The stepwise bead height increase of a step size of approximately $50 \mathrm{~nm}$ after the force jump is from unpairing of the LOV2-SsrA/ $\mathrm{SspB}$ complex. Following the unpairing, the abrupt extension increase and decrease steps with sizes of approximately $15 \mathrm{~nm}$ are from the unfolding and refolding transitions of the $\mathrm{SspB}$ domain (indicated by blue arrows). The brown and dark gray lines show a 20-FFT smooth of the raw data (gray). The inset is an enlargement of the unpairing event in the first cycle. (b) Force-dependent lifetimes of LOV2-SsrA/SspB complex in red light (without FMN in solution, light red) and in blue light (without FMN in solution, light blue; with $2 \mu \mathrm{M}$ FMN, blue). The solid squares indicate the mean lifetime at a force, while the hollow circles represent individual lifetimes measured. Over 80 lifetimes are measured for each force. The horizontal error bars indicate $10 \%$ of relative force calibration uncertainty of the system (see Sec. IV). The vertical error bars indicate standard errors.

at $37^{\circ} \mathrm{C}$ is only slightly weaker than that at $23^{\circ} \mathrm{C}$. It still could withstand forces of several piconewtons over seconds to tens of seconds, suggesting that the iLID-nano system can be used to modulate certain mechanotransduction processes involving similar force and lifetime ranges in the corresponding force-transmission supramolecular linkages at the physiological temperature.
In order to explore the applications of the iLID-nano system to modulate mechanotransduction of cells, we test the system on talin-mediated mechanotransduction at focal adhesion. We express truncated segments of talin-1 molecule, respectively, fused with the dimerizing domains LOV2-SsrA and SspB between talin rod domains 10 and 11 in mouse kidney fibroblast cells with talin-1 and -2 deletion [Figs. 1(a) and 7(c)] [6]. Upon switching on the activating blue light, it is expected that the iLIDinduced dimerization of these two talin segments in cells will result in the reconstitution of a full-length talin 1 to establish a stable force-transmission supramolecular integrin-talin-actin linkage and enable integrin- or talinmediated mechanotransduction of cells at focal adhesions.

Prior to exposure to activating blue light, the cells transfect with the talin-iLID system adhered to a fibronectin-coated cover slip surface through nascent adhesion clusters at the cell periphery and have a round shape consistent with integrin activation by the talin head without connection to F-actin [Fig. 7(d), left] [39]. Upon blue-light illumination, we observe that focal adhesions are formed within a few minutes [Fig. 7(d), right]. The cell shape becomes polarized, and the cell starts to migrate by $10 \mathrm{~min}$ postactivation. A talin C-terminus fragment is recruited to the membrane as observed by an increase in the mEmerald total internal reflection fluorescence (TIRF) signal after activation [Fig. 7(e)]. Interestingly, we find that focal adhesions newly formed after the activation by blue light engage talin $\mathrm{N}$ and $\mathrm{C}$ termini at a similar rate, suggesting that fully reconstituted talin is recruited immediately rather than an initial buildup of talin $\mathrm{N}$ followed by attachment of talin C [Fig. 7(f)]. Upon the cessation of blue-light illumination, the cells become static and either become more symmetric or retract to a much reduced attached area (Supplemental Movie S1 [37]). We also observe that focal adhesions are able to undergo the complete formation, maturation, and disassembly cycle (Supplemental Movie S1 [37]). Furthermore, the talin-mediated focal adhesion dynamics and cell motility could be cyclically modulated by cyclic light switching on and off [Fig. 7(e) and Supplemental Movie S2 [37]]. Together, these results corroborate that talin-1 may be subject to at least several piconewton forces which need to persist for seconds to tens of seconds for its mechanotransduction functions, consistent with the force level and turnover rate of talin estimated from previous studies $[6,13,15]$, and demonstrate that the iLID-nano system can be used to modulate the talinmediated mechanotransduction at focal adhesions.

\section{DISCUSSIONS}

In summary, utilizing a single-molecule constructs design, we directly quantify the mechanical stability of the widely used iLID-nano system and quantitatively show that the mechanical stability of the iLID-nano system meets the physiological requirement for talin-mediated 
(a)

LOV2-SsrA/SspB unpairing

Blue light

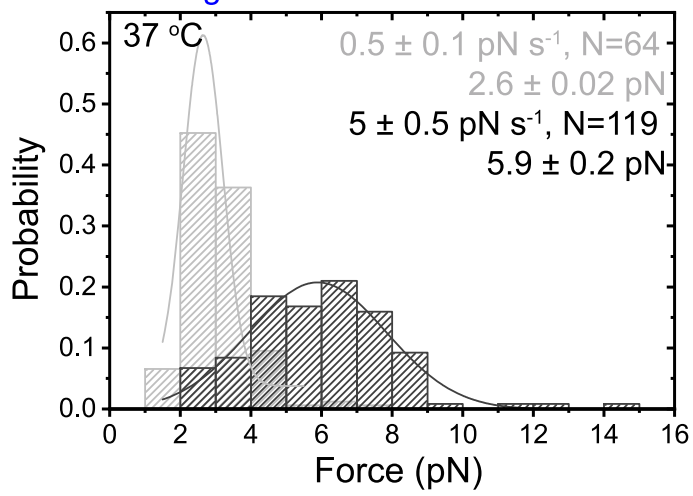

(b)

LOV2-SsrA/SspB

Blue light

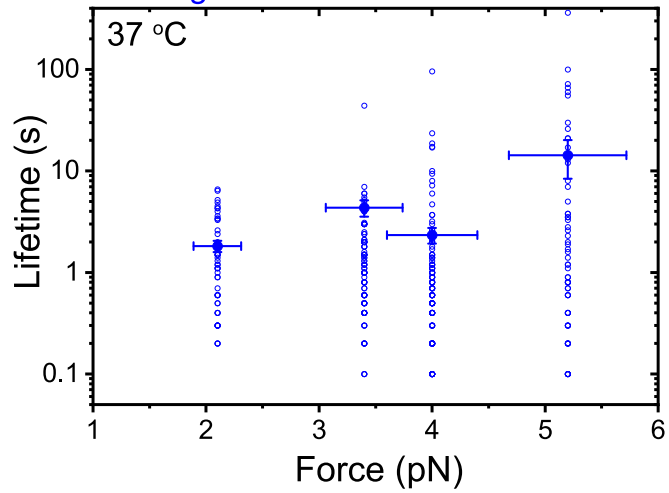

(c)
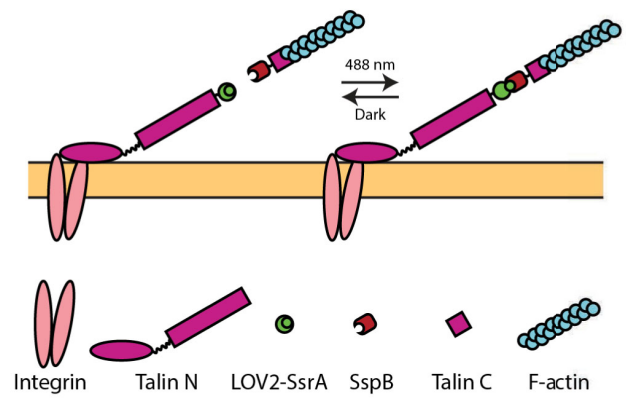

(d)

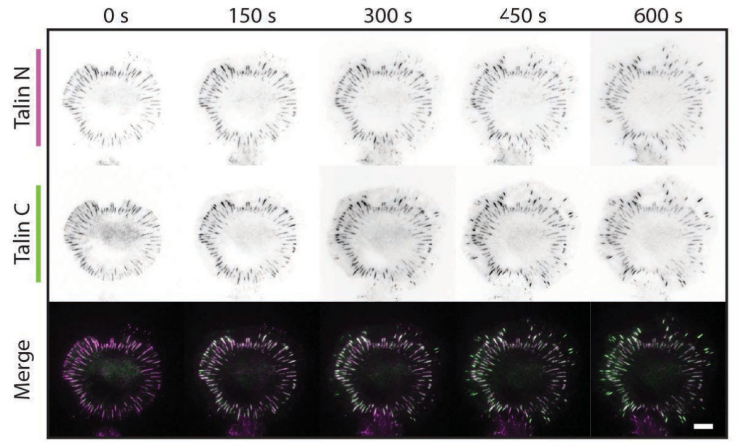

(e)

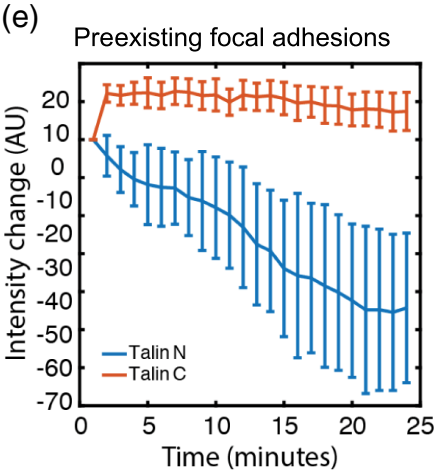

(f)

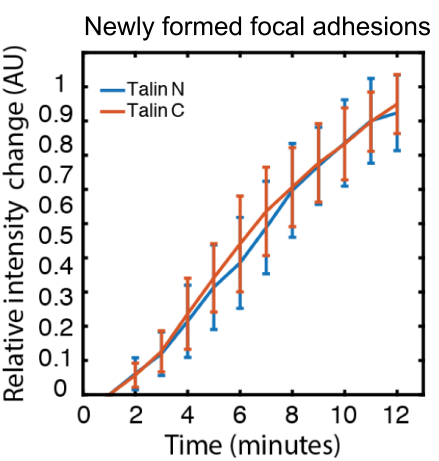

(g)

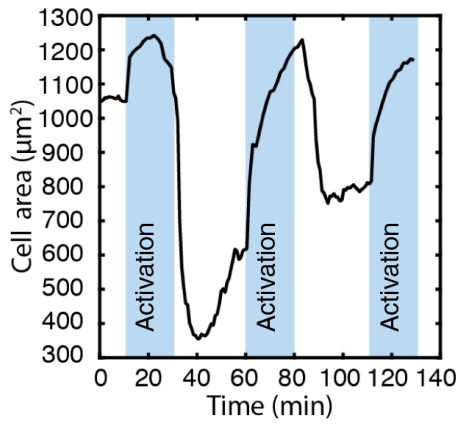

FIG. 7. Modulation of talin-mediated mechanotransduction using iLID-nano. (a) Normalized histograms of LOV2-SsrA/SspB complex unpairing forces obtained at force-loading rates of $0.5 \pm 0.1$ (light gray) and $5 \pm 0.5 \mathrm{pN} \mathrm{s}^{-1}$ (gray) in blue light at $37^{\circ} \mathrm{C}$. The curves are Gaussian fitting curves. (b) Force-dependent lifetimes of LOV2-SsrA/SspB complex in blue light. (a) and (b) are obtained in the absence of FMN in solution. The solid blue squares indicate the mean lifetime at a force, while the hollow blue circles represent individual lifetimes measured. Over 50 lifetimes are measured for each force. The horizontal error bars indicate $10 \%$ of relative force calibration uncertainty of the system (see Sec. IV). The vertical error bars indicate standard errors. (c) Illustration of the modification on the talin-1 protein using iLID-nano. (d) The changes in cell morphology and FA profiles after blue-light switch on for the iLID-nano system. Scale bar, $10 \mu \mathrm{m}$. (e). Engagement of the talin C terminus upon activation of blue light based on ten focal adhesions. (f) Recruitment of talin N and C termini at the same rate in ten new focal adhesions. (g) Change in cell area from Supplemental Movie S2 [37] when exposed to pulsing blue light. Error bars indicate the standard deviation.

mechanotransduction at cell-matrix adhesions $[6,13,15]$. These results suggest that the iLID-nano system can potentially be implemented to temporally and spatially control certain mechanotransduction activities of cells by direct control of force transmission in key force-transmission proteins.
The distinct difference between the mechanical stability of the LOV2 domain with or without FMN in solution suggests that the FMN-bound LOV2 has a much higher mechanical stability than that of the apo state of LOV2. In addition, the results in Fig. 5 show that the pairing between the FMN-bound LOV2-SsrA and $\mathrm{SspB}$ is strongly 
dependent on blue-light activation, in contrast to the constitutively activated pairing between the apo state of LOV2-SsrA and SspB (Fig. S7 [37]). This contrast suggests that the FMN binding to the LOV2 core stabilizes the interaction of the LOV2-SsrA in the dark state, resulting in the suppression of the SsrA-SspB interaction, which can be released by blue light.

The force-dependent lifetime of the LOV2-SsrA/SspB complex shows that it can survive seconds to tens of seconds at forces of several piconewtons over the physiologically relevant temperature range of cells, suggesting that it is suitable to control mechanotransduction involving force-transmission linkages that are subject to similar or less forces and survival timescale in cells, such as talinintegrin-mediated cell adhesion to ECM and cadherincatenin-mediated cell-cell adherence junction. Besides the frequently used iLID-nano system that is quantified in this study, there are a number of other molecular optogenetic switch systems that have been developed in recent years, including iLID-micro, which has a lower pairing affinity than iLID-nano [7], LOVTRAP [23], PhyB/ PIF6 pair [40], Cry2/CIB1 pair [41], FKF/GI pair [42], etc. Whether these molecular optogenetic switch systems can provide sufficient mechanical stability for mechanotransduction studies warrants future quantification and exploration.

Since molecular optogenetic switch systems can be spatially and temporarily activated or deactivated in regions of interest in a cell or an organism using light, their capability of withstanding physiological levels of forces and survival lifetime make them an ideal handle to modulate mechanotransduction via controlling a critical force-bearing protein involved in the corresponding forcetransmission pathway. This capability is demonstrated in this study using a talin-mediated cell spreading and migration assay. Furthermore, by combining multiple orthogonal mechanically stable molecular optogenetic switch systems or combining with mechanically stable chemically induced dimerization systems $[39,43,44]$, more comprehensive and flexible control of the cellular mechanotransduction can be achieved.

An important aspect of cellular mechanotransduction is the capability of cells to sense and respond to the mechanical properties of the surrounding extracellular matrix. To understand how cells sense the mechanical property of the surrounding matrix, hydrogels with different stiffnesses have been developed as important platforms for cell culture systems. It is highly desirable to develop a hydrogel system whose mechanical property can be tuned spatially, temporally, and reversibly. The molecular optogenetic and chemical switch systems provide a potential means for such modulation [45].

Another important potential application of the molecular optogenetic switch systems is the development of novel active matter systems with photoswitchable properties.
Active matter systems are made up of self-driven components which extract energy from their surroundings to generate mechanical work. One important type of active matter systems that is closely relevant to mechanobiology is an active gel consisting of actin or microtubule filaments, cross-linked by passive cross-linkers, contracted by myosin or kinesin motors $[46,47]$. The density, extensibility, and rigidity of the passive cross-linker are a critical determinant of the properties of the active gel. An active gel system with photoswitchable properties will power the studies of the physical principles underlying the active gel's properties. The implementation of molecular optogenetic switch systems in the assembly of active gels is emerging. One example is the just-published work by Ross et al. [48], which reports the use of the iLID system to control the organization and forces in a microtubule- and kinesin-based active gel. For such applications, the quantification of the mechanical stability of a variety of molecular optogenetic switch systems will be critical for a quantitative understanding of the behaviors of active gel systems.

Thus, mechanically stable molecular optogenetic switch systems have a broad range of important applications not only in revealing the molecular mechanisms underlying mechanotransduction of cells, but also in developing novel hydrogels and active matters that can be conveniently controlled with light. The single-molecule-construct assay developed in this study can be utilized for future studies of many other molecular optogenetic switch systems, to build up a toolbox of molecular optogenetic switch systems that have a wide range of mechanical stability for different applications.

\section{METHODS AND MATERIALS}

\section{A. Plasmid constructs and protein expression}

Two plasmids are constructs for single-molecule stretching experiments: (i) pET151-avi-2I27-SsrA-FH1-SspB2I27-spy and (ii) pET151-avi-2I27-LOV2-SsrA-FH1SspB-2I27-spy. Briefly, the DNA fragments encoding LOV2, SsrA, and SspB are amplified by polymerase chain reaction $[7,49]$. The FH1 linker DNA fragment is synthesized by geneArt. The corresponding DNA fragments are then assembled into a pET151-avi-2I27-inset-2I27-spy plasmid [16] by HiFi DNA Assembly (NEB). Each plasmid is cotransformed with a BirA plasmid and expressed in $E$. coli BL21 (DE3) cultured in LB media with D-biotin (Sigma Aldrich) and affinity purified through 6His-tag. More details of plasmid constructs and protein expression is shown in Supplemental Material, Sec. S1 [37].

\section{B. Single-protein manipulation and analysis}

A vertical magnetic tweezers setup is combined with a disturbance-free, rapid solution-exchange flow channel for conducting in vitro protein stretching experiments [33-35]. All in vitro protein stretching experiments are performed in 
solution containing 1X phosphate-buffered saline, 1\% bovine serum albumin, $2 \mathrm{mM}$ Dithiothreitol, $10 \mathrm{mM}$ sodium L-ascorbate at $23^{\circ} \mathrm{C}$, in the presence of ambient light in a typical lab environment with approximately 10 lux. A 470-nm blue LED mounted onto our magnetic tweezers setup is used for blue-light activation (approximately $1 \mathrm{~mW} \times \mathrm{cm}^{-2}$ ) [7]. In the absence of blue-light activation, a 617-nm red LED mounted onto our magnetic tweezers setup is used. The force calibration of the magnetic tweezers setup has a $10 \%$ uncertainty due to the heterogeneity of the diameter of paramagnetic beads [33], and the bead-height determination has an approximately 5-nm uncertainty due to the thermal fluctuation of the tethered bead. The lifetime determination of the complex has an approximately $20 \mathrm{~ms}$ resolution. More details can be found in Supplemental Material, Secs. S2-S4 [37].

\section{Cell culture}

Mouse kidney fibroblast with talin-1 and talin-2 deletion was kindly provided by Dr. Carsten Grashoff (Max Planck Institute of Biochemistry, Martinsreid, Germany) and has been described previously [6]. Cells are cultured in a 5\% $\mathrm{CO}_{2}, 37^{\circ} \mathrm{C}$ humidified atmosphere. The culture media is DMEM media (Life Technologies), supplemented with $10 \%$ Fetal Bovine Serum, sodium pyruvate, and penicillin or streptomycin. For transfection, cells are resuspended at a density of $6 \times 10^{6}$ cells per milliliter and mixed with $0.5-2.0 \mu \mathrm{g}$ of expression vectors per each electroporation reaction. Transfection is performed by electroporation (Neon, Life Technologies) per the manufacturer's protocol. For imaging, transfected cells are sparsely plated at a density of $7000 / \mathrm{cm}^{2}$ on fibronectin-coated cover slips or glass-bottom dishes (Iwaki, Japan). Cells are tested monthly for mycoplasma contamination.

\section{Generation of light-sensitive talin constructs}

All fluorescent protein (FP) fusion constructs are based upon either $\mathrm{N} 1$ or $\mathrm{C} 1$ Clontech-style vectors with $\mathrm{pCMV}$ (Cytomegalovirus) promoters. For pmApple-TalinN10LOV2-SsrA, the construct contains the mApple fluorescent protein at the $\mathrm{N}$ terminus, conjugated with mouse talin1 (residue: 1-1973) and LOV2-SsrA sequence. For SspBTalinC11-mEmerald, the construct contains SspB at the N terminus, conjugated with mouse talin1 (residue: 19742541), followed by mEmerald fluorescent protein at the $C$ terminus. Expression vectors are generated by gene synthesis (Epoch Life Sciences), verified by sequencing, and amplified by QIAgen Miniprep or Maxiprep kits per the manufacturer's protocol.

\section{E. Live-cell TIRF microscopy}

TIRF imaging of live cells is performed using a Nikon Eclipse Ti inverted microscope (Nikon Instruments), equipped with a motorized TIRF illuminator, with a polarization-maintaining optical fiber coupled laser combiner (100 mW $405 \mathrm{~nm}, 60 \mathrm{~mW} 488 \mathrm{~nm}, 50 \mathrm{~mW} 561 \mathrm{~nm}$, and $100 \mathrm{~mW} 642 \mathrm{~nm}$ solid-state lasers, Omicron Laserage), a light-emitting-diode-based epifluorescence excitation source (SOLA, Lumencor), an ORCA-flash $4.0 \mathrm{sCMOS}$ camera (Hamamatsu), a $60 \times$ N.A. 1.49 apo TIRF objective lens (Nikon Instruments), and an Okolab stage-top chamber with $\mathrm{CO} 2$ and temperature control (Okolab, Italy). A 550$\mathrm{nm}$-long pass filter is placed in the differential interference contrast light path to remove activating blue wavelengths. Activation of the iLID system is performed with the $488 \mathrm{~nm}$ laser in the TIRF configuration.

\section{ACKNOWLEDGMENTS}

The authors thank the protein expression core and science communication core of the Mechanobiology Institute. The research is funded by the Singapore Ministry of Education Academic Research Fund Tier 2 (MOE2019-T2-1-099 to J. Y. and P. K.), National Research Foundation, Prime Minister's Office, Singapore, under its NRF Investigatorship Program (NRF Investigatorship Grant No. NRF-NRFI2016-03 to J. Y.), and the Ministry of Education under the Research Centres of Excellence program (to J. Y. and P. K.). M. Y., S. L., P. K., and J. Y. conceived the study. M. Y. and S. L. designed the singlemolecule assay, performed the experiments, and analyzed the data; G.Z., B. S., Z.X., and P. K. performed the cell imaging experiments and data analysis. M. Y., S. L., and J. Y. interpreted the data and wrote the paper.

[1] G. Charras and A. S. Yap, Tensile Forces and Mechanotransduction at Cell-Cell Junctions, Curr. Biol. 28, R445 (2018).

[2] A. W. Orr, B. P. Helmke, B. R. Blackman, and M. A. Schwartz, Mechanisms of Mechanotransduction, Dev. Cell 10, 11 (2006).

[3] M. A. Wozniak, K. Modzelewska, L. Kwong, and P. J. Keely, Focal Adhesion Regulation of Cell Behavior, Biochim. Biophys. Acta 1692, 103 (2004).

[4] G. Giannone, B. J. Dubin-Thaler, H.-G. Döbereiner, N. Kieffer, A. R. Bresnick, and M. P. Sheetz Periodic lamellipodial contractions correlate with rearward actin waves. Cell 116, 431-43 (2004).

[5] C. Grashoff et al., Measuring Mechanical Tension across Vinculin Reveals Regulation of Focal Adhesion Dynamics, Nature (London) 466, 263 (2010).

[6] K. Austen, P. Ringer, A. Mehlich, A. Chrostek-Grashoff, C. Kluger, C. Klingner, B. Sabass, R. Zent, M. Rief, and C. Grashoff, Extracellular Rigidity Sensing by Talin IsoformSpecific Mechanical Linkages, Nat. Cell Biol. 17, 1597 (2015).

[7] G. Guntas, R. A. Hallett, S. P. Zimmerman, T. Williams, H. Yumerefendi, J. E. Bear, and B. Kuhlman, Engineering an Improved Light-Induced Dimer (iLID) for Controlling the 
Localization and Activity of Signaling Proteins, Proc. Natl. Acad. Sci. U.S.A. 112, 112 (2015).

[8] H. K. Song and M. J. Eck, Structural Basis of Degradation Signal Recognition by sspb, a Specificity-Enhancing Factor for the clpxp Proteolytic Machine, Mol. Cell 12, 75 (2003).

[9] B. Zakeri, J. O. Fierer, E. Celik, E. C. Chittock, U. SchwarzLinek, V. T. Moy, and M. Howarth, Peptide Tag Forming a Rapid Covalent Bond to a Protein, through Engineering a Bacterial Adhesin, Proc. Natl. Acad. Sci. U.S.A. 109, E690 (2012).

[10] S. Le, M. Yu, and J. Yan, Phosphorylation Reduces the Mechanical Stability of the $\alpha$-Catenin/ $\beta$-Catenin Complex, Angew. Chem., Int. Ed. Engl. 58, 18663 (2019).

[11] S. Le, M. Yu, and J. Yan, Direct Single-Molecule Quantification Reveals Unexpectedly High Mechanical Stability of Vinculin-Talin/ $\alpha$-Catenin Linkages, Sci. Adv. 5, eaav2720 (2019).

[12] M. Yu, S. Le, Y.-C. Ammon, B. T. Goult, A. Akhmanova, and J. Yan, Force-Dependent Regulation of Talin-Kank1 Complex at Focal Adhesions, Nano Lett. 19, 5982 (2019).

[13] M. Himmel, A. Ritter, S. Rothemund, B. V. Pauling, K. Rottner, A. R. Gingras, and W. H. Ziegler, Control of High Affinity Interactions in the Talin C Terminus, J. Biol. Chem. 284, 13832 (2009).

[14] G. K. Hákonardóttir, P. López-Ceballos, A. D. HerreraReyes, R. Das, D. Coombs, G. Tanentzapf, and V. M. Weaver, In Vivo Quantitative Analysis of Talin Turnover in Response to Force, Mol. Biol. Cell 26, 4149 (2015).

[15] M. Yao, B. T. Goult, B. Klapholz, X. Hu, C. P. Toseland, Y. Guo, P. Cong, M. P. Sheetz, and J. Yan, The Mechanical Response of Talin, Nat. Commun. 7, 11966 (2016).

[16] S. Le et al., Mechanotransmission and Mechanosensing of Human Alpha-Actinin 1, Cell Rep. 21, 2714 (2017).

[17] S. Le, M. Yu, L. Hovan, Z. Zhao, J. Ervasti, and J. Yan, Dystrophin as a Molecular Shock Absorber, ACS Nano 12, 12140 (2018).

[18] S. M. Pang, S. Le, A. V. Kwiatkowski, and J. Yan, Mechanical Stability of $\alpha \mathrm{T}-$ Catenin and Its Activation by Force for Vinculin Binding, Mol. Biol. Cell 30, 1930 (2019).

[19] P. Ringer, A. Weiß1, A.-L. Cost, A. Freikamp, B. Sabass, A. Mehlich, M. Tramier, M. Rief, and C. Grashoff, Multiplexing Molecular Tension Sensors Reveals Piconewton Force Gradient across Talin-1, Nat. Methods 14, 1090 (2017).

[20] H. Liu, X. Yu, K. Li, J. Klejnot, H. Yang, D. Lisiero, and C. Lin, Photoexcited CRY2 Interacts with CIB1 to Regulate Transcription and Floral Initiation in Arabidopsis, Science 322, 1535 (2008).

[21] M. Harterink, P. van Bergeijk, C. Allier, B. de Haan, S. van den Heuvel, C. C. Hoogenraad, and L.C. Kapitein, Light-Controlled Intracellular Transport in Caenorhabditis elegans, Curr. Biol. 26, R153 (2016).

[22] G. Guglielmi, H. J. Falk, and S. D. Renzis, Optogenetic Control of Protein Function: From Intracellular Processes to Tissue Morphogenesis, Trends Cell Biol. 26, 864 (2016).

[23] H. Wang, M. Vilela, A. Winkler, M. Tarnawski, I. Schlichting, H. Yumerefendi, B. Kuhlman, R. Liu, G. Danuser, and K. M. Hahn, LOVTRAP: An Optogenetic System for Photoinduced Protein Dissociation, Nat. Methods 13, 755 (2016).

[24] M. Adrian, W. Nijenhuis, R. I. Hoogstraaten, J. Willems, and L.C. Kapitein, A Phytochrome-Derived Photoswitch for Intracellular Transport, ACS Synth. Biol. 6, 1248 (2017).

[25] L. Valon, A. Marín-Llauradó, T. Wyatt, G. Charras, and X. Trepat, Optogenetic Control of Cellular Forces and Mechanotransduction, Nat. Commun. 8, 14396 (2017).

[26] Z. Liao, A. Kasirer-Friede, and S. J. Shattil, Optogenetic Interrogation of Integrin $\alpha \mathrm{V} \beta 3$ Function in Endothelial Cells, J. Cell Sci. 130, 3532 (2017).

[27] R. Jöhr, M. S. Bauer, L. C. Schendel, C. Kluger, and H. E. Gaub, Dronpa: A Light-Switchable Fluorescent Protein for Opto-biomechanics, Nano Lett. 19, 3176 (2019).

[28] A. Losi, E. Polverini, B. Quest, and W. Gärtner, First Evidence for Phototropin-Related Blue-Light Receptors in Prokaryotes, Biophys. J. 82, 2627 (2002).

[29] J. P. Zayner, C. Antoniou, and T. R. Sosnick, The AminoTerminal Helix Modulates Light-Activated Conformational Changes in aslov2, J. Mol. Biol. 419, 61 (2012).

[30] M. Dorn, M. Jurk, A. Wartenberg, A. Hahn, and P. Schmieder, Lov Takes a Pick: Thermodynamic and Structural Aspects of the Flavin-Lov-Interaction of the Blue-Light Sensitive Photoreceptor ytva from Bacillus subtilis, PLoS One 8, e81268 (2013).

[31] J. P. Zayner and T. R. Sosnick, Factors That Control the Chemistry of the Lov Domain Photocycle, PLoS One 9, e87074 (2014).

[32] M. Yu, S. Le, A. K. Efremov, X. Zeng, A. Bershadsky, and J. Yan, Effects of Mechanical Stimuli on Profilin- and ForminMediated Actin Polymerization, Nano Lett. 18, 5239 (2018).

[33] H. Chen, H. Fu, X. Zhu, P. Cong, F. Nakamura, and J. Yan, Improved High-Force Magnetic Tweezers for Stretching and Refolding of Proteins and Short DNA, Biophys. J. 100, 517 (2011).

[34] S. Le, R. Liu, C. T. Lim, and J. Yan, Uncovering Mechanosensing Mechanisms at the Single Protein Level Using Magnetic Tweezers, Methods 94, 13 (2016).

[35] S. Le et al., Disturbance-Free Rapid Solution Exchange for Magnetic Tweezers Single-Molecule Studies, Nucl. Acids Res. 43, e113 (2015).

[36] X. Zhao, X. Zeng, C. Lu, and J. Yan, Studying the Mechanical Responses of Proteins Using Magnetic Tweezers, Nanotechnology 28, 414002 (2017).

[37] See Supplemental Material at http://link.aps.org/ supplemental/10.1103/PhysRevX.10.021001 for Supplemental Figures, captions of Supplemental Movies, details of protein sequences, and data analysis.

[38] J. F. Marko and E. D. Siggia, Stretching DNA, Macromolecules 28, 8759 (1995).

[39] Y. Wang, S. F. H. Barnett, S. Le, Z. Guo, X. Zhong, P. Kanchanawong, and J. Yan, Label-Free Single-Molecule Quantification of Rapamycin-Induced fkbp-frb Dimerization for Direct Control of Cellular Mechanotransduction, Nano Lett. 19, 7514 (2019).

[40] C. E. Buckley, R. E. Moore, A. Reade, A. R. Goldberg, O. D. Weiner, and J. D. W. Clarke, Reversible Optogenetic Control of Subcellular Protein Localization in a Live Vertebrate Embryo, Dev. Cell 36, 117 (2016).

[41] L. Duan, J. Hope, Q. Ong, H.-Y. Lou, N. Kim, C. McCarthy, V. Acero, M.Z. Lin, and B. Cui, Understanding CRY2 
Interactions for Optical Control of Intracellular Signaling, Nat. Commun. 8, 547 (2017).

[42] M. Yazawa, A. M. Sadaghiani, B. Hsueh, and R. E. Dolmetsch, Induction of Protein-Protein Interactions in Live Cells Using Light, Nat. Biotechnol. 27, 941 (2009).

[43] S. Voß, L. Klewer, and Y.-W. Wu, Chemically Induced Dimerization: Reversible and Spatiotemporal Control of Protein Function in Cells, Curr. Opin. Chem. Biol. 28, 194 (2015).

[44] D. Kostrz, H. K. Wayment-Steele, J. L. Wang, M. Follenfant, V. S. Pande, T. R. Strick, and C. Gosse, A Modular DNA Scaffold to Study Protein-Protein Interactions at SingleMolecule Resolution, Nat. Nanotechnol. 14, 988 (2019).

[45] H. Nakamura et al., Intracellular Production of Hydrogels and Synthetic RNA Granules by Multivalent Molecular Interactions, Nat. Mater. 17, 79 (2018).
[46] T. Sanchez, D. T. N. Chen, S. J. DeCamp, M. Heymann, and Z. Dogic, Spontaneous Motion in Hierarchically Assembled Active Matter, Nature (London) 491, 431 (2012).

[47] D. Mizuno, C. Tardin, C. F. Schmidt, and F. C. Mackintosh, Nonequilibrium Mechanics of Active Cytoskeletal Networks, Science 315, 370 (2007).

[48] T. D. Ross, H. J. Lee, Z. Qu, R. A. Banks, R. Phillips, and M. Thomson, Controlling Organization and Forces in Active Matter through Optically Defined Boundaries, Nature (London) 572, 224 (2019).

[49] O. I. Lungu, R. A. Hallett, E. J. Choi, M. J. Aiken, K. M. Hahn, and B. Kuhlman, Designing Photoswitchable Peptides Using the AsLOV2 Domain, Chem. Biol. 19, 507 (2012). 